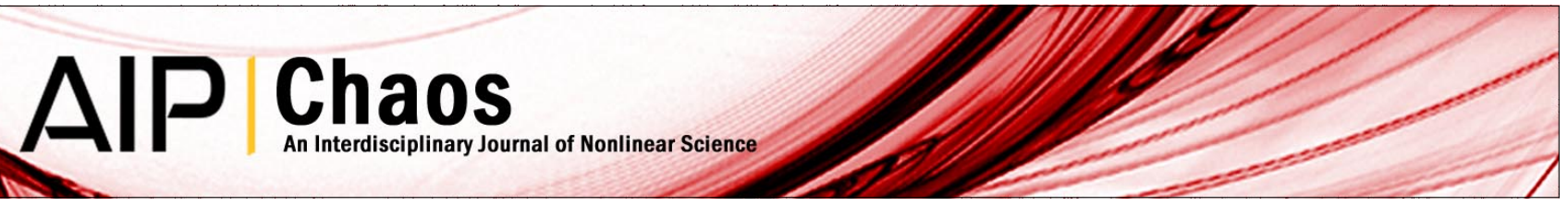

\title{
Edge state and crisis in the Pierce diode
}

Pablo R. Muñoz, Joaquim J. Barroso, Abraham C.-L. Chian, and Erico L. Rempel

Citation: Chaos 22, 033120 (2012); doi: 10.1063/1.4736860

View online: http://dx.doi.org/10.1063/1.4736860

View Table of Contents: http://chaos.aip.org/resource/1/CHAOEH/v22/i3

Published by the American Institute of Physics.

\section{Related Articles}

Influence of wall thickness on the stability of the resistive wall mode in tokamak plasmas

Phys. Plasmas 20, 012504 (2013)

Surface loss probability of $\mathrm{H}$ radicals on silicon thin films in $\mathrm{SiH} 4 / \mathrm{H} 2$ plasma

J. Appl. Phys. 113, 013303 (2013)

Formation of surface nano-structures by plasma expansion induced by highly charged ions

Phys. Plasmas 19, 123510 (2012)

New dual gas puff imaging system with up-down symmetry on experimental advanced superconducting tokamak Rev. Sci. Instrum. 83, 123506 (2012)

Ablation driven by hot electrons generated during the ignitor laser pulse in shock ignition

Phys. Plasmas 19, 122705 (2012)

\section{Additional information on Chaos}

Journal Homepage: http://chaos.aip.org/

Journal Information: http://chaos.aip.org/about/about_the_journal

Top downloads: http://chaos.aip.org/features/most_downloaded

Information for Authors: http://chaos.aip.org/authors

\section{ADVERTISEMENT}

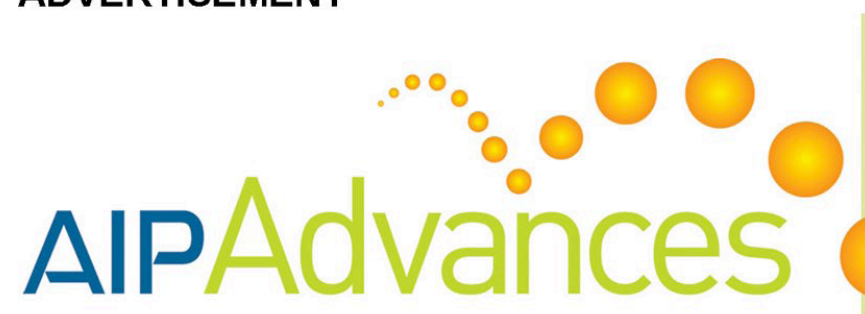

Explore AIP's new open-access journal

Article-level metrics now available

Join the conversation! Rate \& comment on articles 


\title{
Edge state and crisis in the Pierce diode
}

\author{
Pablo R. Muñoz, ${ }^{1, \text { a) }}$ Joaquim J. Barroso, ${ }^{2}$ Abraham C.-L. Chian, ${ }^{2,3}$ and Erico L. Rempel ${ }^{1}$ \\ ${ }^{1}$ Institute of Aeronautical Technology (ITA) and World Institute for Space Environment Research (WISER), \\ CTA/ITA/IEFM, São José dos Campos-SP 12228-900, Brazil \\ ${ }^{2}$ National Institute for Space Research (INPE) and World Institute for Space Environment Research (WISER), \\ P. O. Box 515, São José dos Campos-SP 12227-010, Brazil \\ ${ }^{3}$ Observatoire de Paris, LESIA, CNRS, 92195 Meudon, France
}

(Received 23 November 2011; accepted 27 June 2012; published online 7 August 2012)

\begin{abstract}
We study the chaotic dynamics of the Pierce diode, a simple spatially extended system for collisionless bounded plasmas, focusing on the concept of edge of chaos, the boundary that separates transient from asymptotic dynamics. We fully characterize an interior crisis at the end of a periodic window, thereby showing direct evidence of the collision between a chaotic attractor, a chaotic saddle, and the edge of chaos, formed by a period-3 unstable periodic orbit and its stable manifold. The edge of chaos persists after the interior crisis, when the global attractor of the system increases its size in the phase space. (C) 2012 American Institute of Physics. [http://dx.doi.org/10.1063/1.4736860]
\end{abstract}

A chaotic plasma device, the Pierce diode, is studied using a novel concept from the dynamical systems approach: the edge of chaos. We focus on a periodic window in the parameter space, where typical phenomena such as chaotic transients and interior crisis are observed. In general, chaotic transients arise due to the presence of a surrounding chaotic saddle, and the ensuing interior crisis triggered by the collision between a chaotic attractor and a mediating unstable periodic orbit (UPO). Our results show that the mediating UPO coincides with the edge state that lies in the boundary defined by the edge of chaos. For the first time the interior crisis in the classical Pierce diode is fully characterized. Our results and the methodology developed herein can be used for the characterization of chaotic transitions in other spatially extended systems.

\section{INTRODUCTION}

In a dynamical system, the basin of attraction of a given attractor is the set of initial conditions that converge to that attractor. If the system has more than one attractor, we can define a region that separates the basins: the basin boundary. An extension of this concept was introduced by Skufca et $a l .{ }^{1}$ while studying transition to turbulence in shear flows using a nine-dimensional truncation of the Navier-Stokes equation. In that model, the system has one attractor, coexisting with a chaotic saddle, the structure responsible for the chaotic transients. $^{2-4}$ Skufca et al. ${ }^{1}$ observed that even though only one basin of attraction is present, the phase space can be divided into two regions, depending on whether an initial condition displays a chaotic transient behavior or not. The boundary between these two regions is called the edge of chaos. In the last years, the dynamical properties of the edge of chaos have been studied in a wide variety of applications, such as direct numerical simulations of pipe flow, ${ }^{5}$ a numerical MHD simulation for two-dimensional

\footnotetext{
${ }^{\text {a)} E l e c t r o n i c ~ m a i l: ~ p a b l o c u s @ g m a i l . c o m . ~}$
}

magnetic reconnection, ${ }^{6}$ and a generic two-dimensional map. ${ }^{7}$ These works illustrate the rich dynamical behavior of the edge of chaos and its important role in transitions to chaos and turbulence.

\section{PIERCE DIODE}

In this work we apply ideas of Section I to the Pierce diode, a one-dimensional spatially extended plasma model. Constituting the simplest model for collisionless bounded plasma systems, the classical Pierce diode ${ }^{8-11}$ is a onedimensional electrostatic parallel-plane diode with gap spacing $L$ into which a monoenergetic electron beam at constant velocity $v_{0}$ and charge density $\rho_{0}$ is injected. An immobile neutralizing ion background with density $\rho_{0}$ is present between the planar electrodes held at the same potential (short-circuit condition). Figure 1 is a schematic representation of this system. In particular, the presence of a controlled amount of background ions inside microwave tubes allows plasma-filled devices to operate at currents much higher than the maximum current for vacuum tubes, thereby increasing significantly the power handling capabilities of microwave tubes. ${ }^{8,12}$ In this context, plasma-assisted devices can be configured as a slow-wave oscillator (PASATRON), backwardwave oscillator (BWO), and a traveling wave amplifier (TWT) operating without focusing magnetic fields. ${ }^{13-15}$ These devices constitute unique sources of microwave radiation, in which the beam propagation in the absence of external magnetic fields is provided by the ion focusing and the electron interaction with the electromagnetic fields. Also, Pierce-type plasma diodes with a background of mobile ions can generate microwaves in a sequence of chaotic pulses whose duration is controlled by the retarding potential and the kind of ionized gas. ${ }^{16}$ From the emitter (at $x=0$ ), the monoenergetic electron beam, after crossing the gap spacing between the plates, is completely absorbed by the collector at $x=L$. Although rather simple, this distributed model exhibits many features of the electron beam dynamics in a variety of microwave electronic devices such as the klystron and the virtual cathode oscillator. ${ }^{12}$ In addition, this model is 


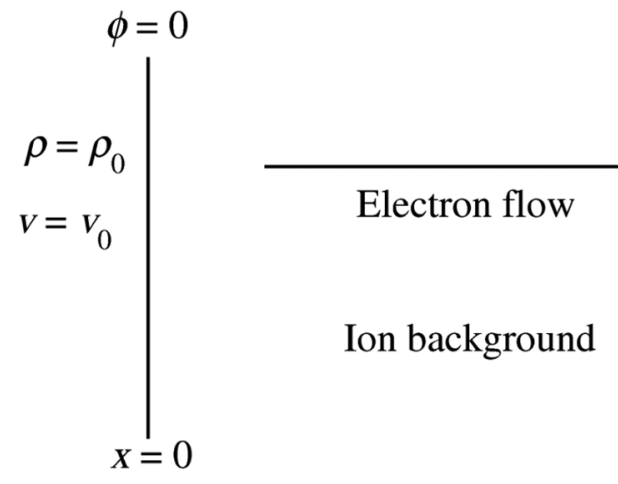

Cathode

FIG. 1. Schematic diagram of the Pierce diode.

used for studying the stability of electron flows in plasmafilled diodes and charge neutralized ion beam transport for inertial confinement fusion ${ }^{17}$ and also space physics applications such as double layers in the magnetospheric cusp. ${ }^{18}$

The system is characterized by the single control parameter $\alpha=\omega_{p} L / v_{0}$, often referred to as the Pierce parameter, where $\omega_{p}=\sqrt{\rho_{0} e / \epsilon_{0} m_{e}}$ denotes the plasma frequency of the electron beam, with $\epsilon_{0}$ the vacuum permittivity and $e$ and $m_{e}$ the electronic charge and the electron mass, respectively. The electron flow in this system can be described by the cold fluid equations, namely, continuity, momentum, and the Poisson's equations

$$
\begin{aligned}
& \frac{\partial \rho}{\partial t}+\frac{\partial(\rho v)}{\partial x}=0, \\
& \frac{\partial v}{\partial t}+v \frac{\partial v}{\partial x}=\frac{\partial \phi}{\partial x}, \\
& \frac{\partial^{2} \phi}{\partial x^{2}}=\alpha^{2}(\rho-1),
\end{aligned}
$$

where $\rho$ is the mass density, $v$ is the electron flow velocity, and $\phi$ is the electric potential. In Eqs. (1)-(3) nondimensional variables (density $\rho$, velocity $v$, electric potential $\phi$, space coordinate $x$, and time $t$ ) are used. ${ }^{19}$ They are related to the corresponding dimensional variables as follows:

$$
\begin{gathered}
\rho^{\prime}=\rho_{0} \rho, \quad v^{\prime}=v_{0} v, \quad \phi^{\prime}=\left(v_{0}^{2} / e\right) \phi, \\
x^{\prime}=L x, \quad t^{\prime}=\left(L / v_{0}\right) t,
\end{gathered}
$$

where the primed variables denote the dimensional values. Then, the boundary conditions are $\rho(0, t)=1, v(0, t)=1$, and $\phi(0, t)=\phi(1, t)=0$.

A linear approximation analysis ${ }^{8,10}$ can be performed on the assumption of solutions of the form $\rho(x, t)=\rho_{0}+\rho_{1}(x, t)$ and $v(x, t)=v_{0}+v_{1}(x, t)$, where time and spatial dependence of $\rho_{1}(x, t)$ and $v_{1}(x, t)$ are, respectively, of the form $e^{-i \omega t}$ and $e^{i k x}$. Substituting these ansatz solutions in Eqs. (1)-(3) and taking just leading terms, we obtain the a linear dispersion relation given by

$$
\begin{aligned}
& 2 \Omega^{2}\left(\Omega^{2}-\alpha^{2}\right)+i \alpha\left\{(\Omega+\alpha)^{2}\left[e^{i(\Omega-\alpha)}-1\right]\right. \\
& \left.-(\Omega-\alpha)^{2}\left[e^{i(\Omega+\alpha)}-1\right]\right\}=0
\end{aligned}
$$

where we define $\Omega$ as a scaled complex frequency

$$
\Omega=\frac{L \omega}{v_{0}}=r+i s .
$$

The dispersion relation (5) provides time growing (unstable) non-oscillatory solutions for $(2 n-1) \pi \leq \alpha \leq 2 \pi n$, $(n=1,2,3, \ldots)$, growing (unstable) oscillatory solutions for $2 \pi n \leq \alpha \leq\left(2 n+1-\epsilon_{n}\right) \pi$, and damped (stable) oscillatory solutions for $\left(2 n-1-\epsilon_{n}\right) \pi \leq \alpha \leq(2 n-1) \pi$, where $0<\epsilon_{n} \ll 1$ varies slightly with $\mathrm{n}$. Thus the stability character of linear oscillatory solutions alternates as the parameter $\alpha$ is increased by $\pi$, as illustrated in Barroso et al. ${ }^{17}$ Then an otherwise single damped mode starts growing at $\alpha=\pi$ and remains unstable until $\alpha=2 \pi$, a situation in which a virtual cathode is formed with electrons being reflected back to the emitter. Moreover, the transition from instability to stability just below each odd multiple of $\pi$ is described by a Hopf bifurcation. ${ }^{10}$

\section{BIFURCATION DIAGRAM}

We solve the continuity and momentum equations (1)-(3) using a first-order backward difference scheme in space and a second-order implicit scheme in time, over a spatial grid with $N=512$ points, using a time step $\Delta t=0.001$ which guarantees the Courant condition is satisfied all the time. In order to satisfy the zero-potential conditions, the Poisson's equation is solved by means of the sine fast Fourier transform method. ${ }^{20}$ The state of the system at each discrete time $t_{k}$ is given by $\rho_{i}^{k}=\rho\left(x_{i}, t_{k}\right)$ and $v_{i}^{k}=v\left(x_{i}, t_{k}\right)$, where $x_{i}$ is a grid point. We define a Poincaré map as $\rho(x=0.25, t)$ $=1$ and $\partial_{t} \rho(x=0.25, t)<0$ to construct a bifurcation diagram by varying the parameter $\alpha$. For every value of $\alpha$, we discard the initial transient (100 iterations) and plot the next 200 iterations of the map. We are interested in a periodic window of period-3 ( $p-3)$ near $\alpha=3 \pi$. Figure 2(a) shows the bifurcation diagram of this periodic window, and Fig. 2(b) the first and second Lyapunov exponents of the attractor using the method by Benettin et al. ${ }^{21}$ In this method, two trajectories $\mathbf{y}(t)$ and $\mathbf{y}(t)+\delta(t)$ are integrated from time $t$ to $t+\Delta t$, and the local separation (or contraction) rate is obtained as

$$
\lambda_{t}=\frac{1}{\Delta t} \ln \frac{|\delta(t+\Delta t)|}{|\delta(t)|} .
$$

Performing the normalization

$$
\delta(t+\Delta t) \mapsto \delta(t+\Delta t) \frac{|\delta(0)|}{|\delta(t+\Delta t)|}
$$

and repeating the integration $M$ times, the maximum Lyapunov exponent is given by the average

$$
\lambda_{1}=\frac{1}{M} \sum_{k=1}^{M} \lambda_{t_{k}} .
$$

The second Lyapunov exponent can be obtained straightforwardly by integrating a third initial condition forming an orthogonal base with $\mathbf{y}$ and $\mathbf{y}+\delta$. 

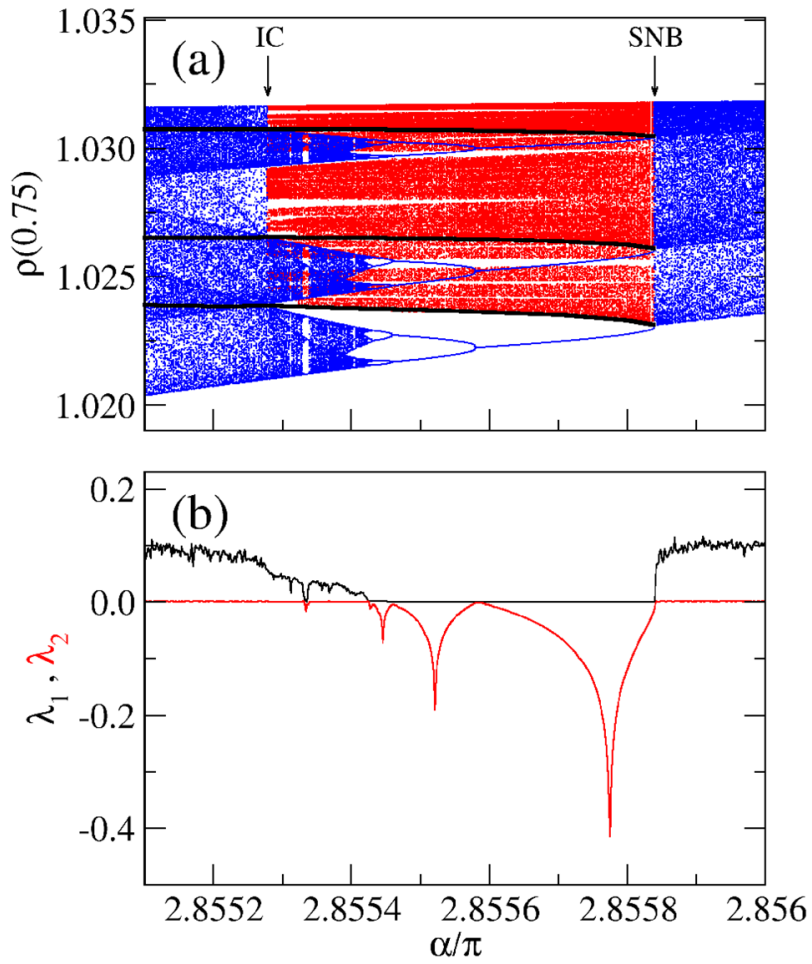

FIG. 2. (a) Bifurcation diagram: a $p-3$ periodic window of the Pierce diode. Within the window the attractor (blue dots) coexists with the surrounding chaotic saddle (red dots) and a $p$-3 UPO (black lines). SNB (IC) denotes saddle-node bifurcation (interior crisis). (b) First (black) and second (red) Lyapunov exponents.

Three types of bifurcation that characterize the periodic window are noteworthy: a saddle-node bifurcation (SNB) at $\alpha \approx 2.85584 \pi$, where a splitting pair of $p$ - 3 stable and unstable periodic orbits arises; a period-doubling cascade leading to a banded chaotic attractor (BCA); and an interior crisis (IC) at $\alpha=\alpha_{\mathrm{c}} \approx 2.8552792 \pi$, where the banded chaotic attractor is converted into a banded chaotic saddle (BCS), and the size of the chaotic attractor is increased. Coexisting with a banded attractor inside the periodic window, a surrounding chaotic saddle (SCS), given by red dots in Fig. 2(a), is responsible for chaotic transients that mimic the dynamics of the larger chaotic attractor outside the periodic window.

\section{LIFETIME FUNCTION AND BISECTION METHOD}

To study how the surrounding chaotic saddle SCS shapes the phase space within the periodic window of Fig. 2, we introduce the lifetime function ${ }^{1}$ of an initial condition

$$
\mathbf{y}_{0}=\left\{\rho_{i}^{0}, v_{i}^{0}\right\}, \quad i=1, \ldots, N,
$$

where $\rho_{i}^{0}$ and $v_{i}^{0}$ are the initial density and velocity profiles in the spatial grid. The lifetime is defined as the time initial condition $\mathbf{y}_{0}$ takes to converge to the attractor inside the periodic window. We use a two-dimensional projection of the Poincaré map $\mathbf{z}_{k}=\left\{\rho\left(x=0.5, t_{k}\right), \rho\left(x=0.75, t_{k}\right\}\right.$ to facilitate the definition of convergence. First, we collect a set of $M$ Poincaré points $S=\left\{\mathbf{z}_{A}^{j}, j=1, \ldots, M\right\}$ in the attractor. Then, we integrate the initial condition $\mathbf{y}_{0}$ generating the discrete two-dimensional Poincaré map $\mathbf{z}_{k}$. For each discrete time step $k$, we define the distance between $\mathbf{z}_{k}$ and $S$ as

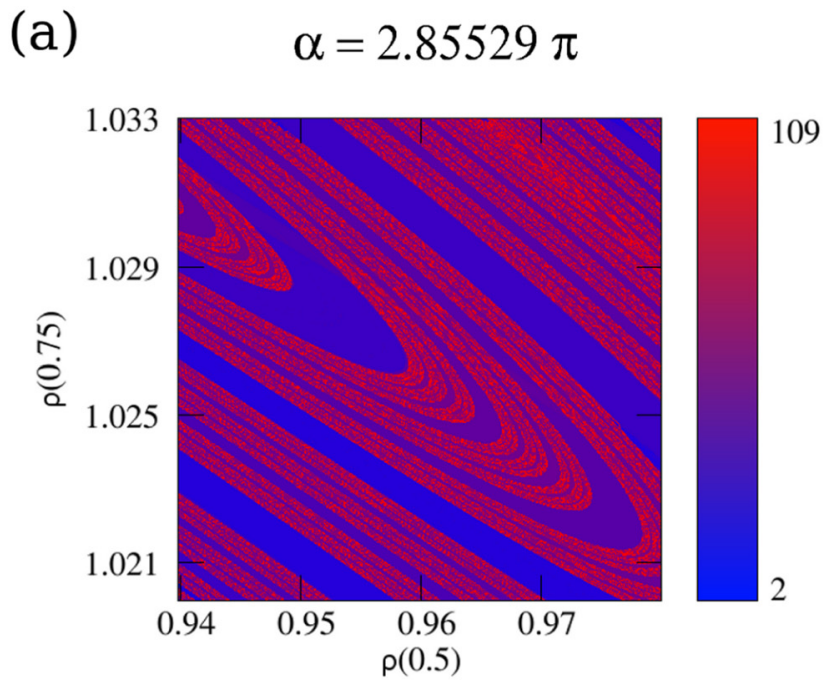

(b)

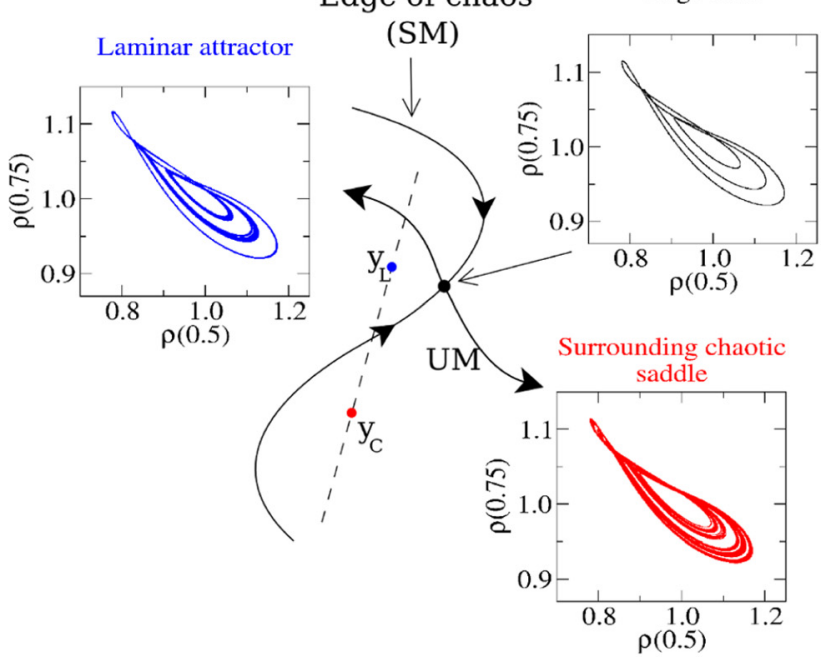

FIG. 3. (a) Density plot of the lifetime, given in units of Poincaré points, in a two-dimensional phase-space projection at $\alpha=2.85529 \pi$. Blue areas indicate initial conditions that converge quickly to the attractor. Initial conditions leading to longer lifetimes are represented by red tones. The edge of chaos is given by the boundary between the blue and red areas. (b) Schematic representation of the edge of chaos (SM) indicated by a solid line and its associated saddle object (the edge state). Any initial condition lying on the edge of chaos will converge to the edge state.

$$
\Delta\left(\mathbf{z}_{k}, S\right)=\min \left(\left\|\mathbf{z}_{k}-\mathbf{z}_{A}^{j}\right\|, \quad j=1, \ldots, M\right),
$$

where $\left\|\mathbf{z}_{k}-\mathbf{z}_{A}^{j}\right\|$ is the Euclidean distance. When the distance to the attractor is less than some suitable threshold $\Delta\left(\mathbf{y}_{k}, S\right)<\delta$, we consider $\mathbf{y}_{k}$ has converged to the attractor. In this case we use $\delta=10^{-4}$. A two-dimensional density plot of the lifetime in the phase-space at $\alpha=2.85529 \pi$ is shown in Fig. 3(a). Blue areas denote initial conditions that converge quickly to the banded chaotic attractor. Areas of longer lifetime, in red tones, exhibit an apparent fractal structure, which indicates the proximity of the corresponding initial conditions to the stable manifold of the surrounding chaotic saddle. Thus, there exist two possible trajectories for a given initial condition in the phase space: (i) the trajectory may converge directly to the attractor or (ii) the trajectory may visit the vicinity of the surrounding 
chaotic saddle before converging to the attractor. Under this circumstance, we can define two regions or pseudobasins of attraction in the phase space: the laminar basin (blue region) related to (i) and the chaotic basin (red region) related to (ii). The basin boundary separating laminar and chaotic basins is the edge of chaos. ${ }^{1}$ The asymptotic trajectory on this boundary is called the edge state (ES). In order to determine the ES, we apply the bisection method. ${ }^{1}$ From Fig. 3(a) we note that any path connecting the two basins must intersect the edge of chaos (dashed line in Fig. 3(b)). First we select two initial conditions, $\mathbf{y}_{L}$ and $\mathbf{y}_{C}$, in the laminar and chaotic basins, respectively. A condition $\mathbf{y}_{C}$ is in the chaotic basin if it has a long lifetime. Such a condition can be obtained as a small perturbation of the unstable steady state $\rho(x, 0)=1+\delta \rho(x)$ and $v(x, 0)=1+\delta v(x)$, with $\delta \rho(0)=\delta v(0)=0$ to satisfy the boundary conditions. The laminar condition $\mathbf{y}_{L}$ can be any point in the attractor. We integrate the initial condition $\mathbf{y}_{M}=\left(\mathbf{y}_{L}+\mathbf{y}_{C}\right) / 2$ and decide which side the initial condition is on. By successive bisections, we reduce the distance $d=\left\|\mathbf{y}_{L}-\mathbf{y}_{C}\right\|$ and bring $\mathbf{y}_{L}$ and $\mathbf{y}_{C}$ close to the edge of chaos. By integrating the system using the final $\mathbf{y}_{L}$ and $\mathbf{y}_{C}$ as initial conditions we generate trajectories that follow the edge of chaos, spend some time near the ES, and then diverge either to the attractor or to the chaotic saddle.

As an example, we apply the bisection method for $\alpha=2.85564 \pi$, in the middle of the periodic window, where the attractor is a $p-3$ stable periodic orbit. Figure 4 shows the Poincaré points of every three iterations of laminar (blue circles) and chaotic (red triangles) initial conditions. Both trajectories follow the edge of chaos in the beginning, passing near the edge state, after which they separate. The laminar trajectory converges quickly and smoothly to the attractor, whereas the chaotic one spends some time near the surrounding chaotic saddle before converging to the attractor. We compute the ES for several values of the control parameter $\alpha$ in the periodic window and conclude that the saddle object that separates the laminar and chaotic basins is the $p-3$ UPO that arises jointly with the $p-3$ periodic attractor at the SNB (black line in Fig. 2(a)). Hence, the edge state is the $p-3$ UPO and its stable manifold (SM) is the edge of chaos.



FIG. 4. Poincaré time series of two trajectories on the laminar side (blue circles) and chaotic side (red triangles) of the edge of chaos before converging to the $p-3$ periodic attractor for $\alpha=2.85564 \pi$. Poincaré points are plotted for each three iterations, $m=1,4,7, \ldots$

\section{INTERIOR CRISIS}

In the following, we examine the role of the edge state in the interior crisis which occurs at the end of the periodic window (Fig. 2). In chaotic systems with one positive Lyapunov exponent, an interior crisis is a sudden transition triggered by a collision between a chaotic attractor, a mediating unstable periodic orbit UPO and its stable manifold. ${ }^{22}$ Moreover, at the onset of crisis, the SCS also collides with the attractor. ${ }^{23}$ One has to find all these structures to characterize the crisis. When the crisis takes place, at $\alpha=\alpha_{c}$, we numerically find the chaotic attractor, the chaotic saddle SCS using the sprinkler method, ${ }^{24}$ and the edge state ES with the bisection method. ${ }^{1}$ We use the fact observed by Rempel et al. ${ }^{2}$ that the boundary of the stable manifold of SCS approximates the stable manifold of the mediating UPO. We compute the stable manifold of the SCS with the projection technique developed by Rempel et al. ${ }^{2}$ to study chaotic transitions in high-dimensional systems. A suitable grid of chosen initial conditions is constructed using one point $A$ from BCA and three points $B, C$, and $D$ from SCS at the vicinity of the collision. Figure 5 shows a threedimensional projection of this grid (grey points) jointly with the attractor BCA (blue) and the chaotic saddle SCS (red) at the moment of the interior crisis. Figure 6(a) shows the attractor BCA (blue), the surrounding chaotic saddle SCS (red dot), and the $p-3$ edge state ES (black crosses) in a two-dimensional projection of the phase space. The grid of initial conditions near the collision corresponds to the dashed rectangle of Fig. 6(a). Figure 6(b) shows an enlargement of this region to elucidate the collision that characterizes the interior crisis. At the onset of crisis, the chaotic saddle (red) and the boundary of its stable manifold (grey) collide with the banded chaotic attractor (blue). The edge state (black cross) and its stable manifold (dashed lines) form the boundary between the attractor and the chaotic saddle pseudo-basins (blue and red regions, respectively, in

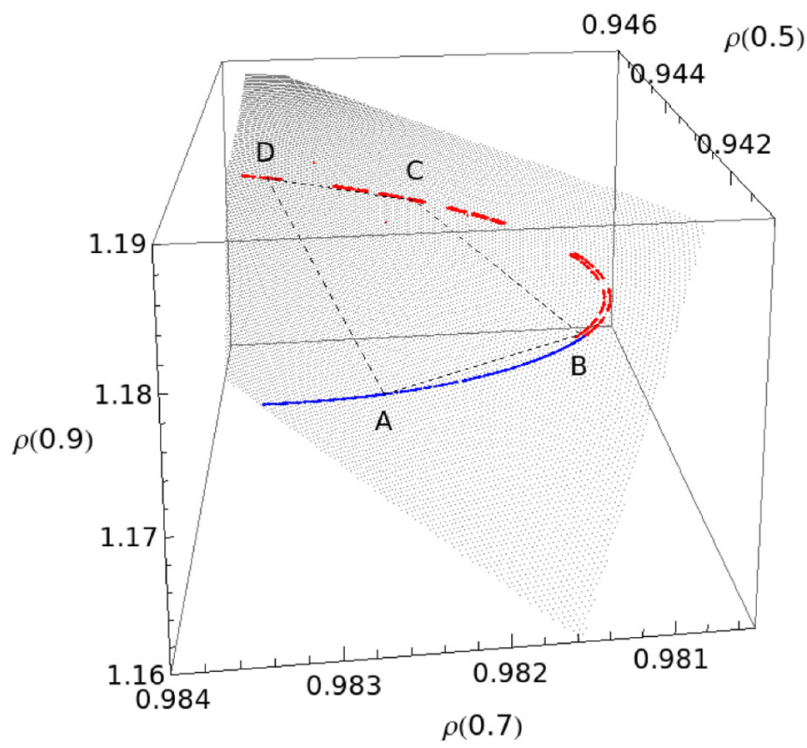

FIG. 5. Three-dimensional projection of the grid of initial conditions generated from four points $A, B, C$ and $D$, containing part of the banded chaotic attractor BCA (blue) and the chaotic saddle SCS (red). 

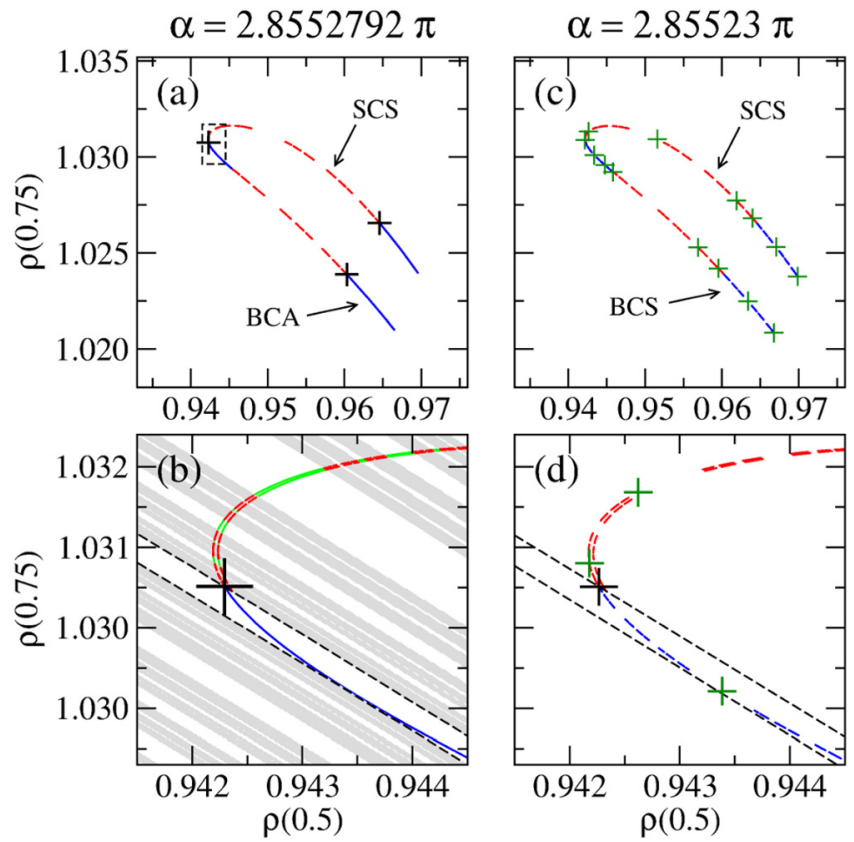

FIG. 6. Two-dimensional Poincaré plots at the onset of ((a) and (b)) and after ((c) and (d)) the interior crisis. (a) Three structures involved at the onset of crisis: the banded chaotic attractor (BCA, blue), the surrounding chaotic saddle (SCS, red) and the $p-3$ edge state (black crosses). (b) An enlargement of the dashed rectangle region indicated in (a). The $p-3$ mediating UPO (the edge state) and its stable manifold (the edge of chaos, dashed line) collide with BCA and SCS. The stable (grey) and unstable (green) manifolds of SCS are also shown. (c) Post-crisis banded chaotic saddle (BCS, blue), SCS, and a p-14 coupling UPO (green crosses) with its branches in the gaps of both banded and surrounding regions. (d) An enlargement of (c). The edge state (black cross) and the edge of chaos (dashed lines) are also shown.

Fig. 3(a)), and when they collide, the edge of chaos also collides simultaneously with them.

\section{CRISIS-INDUCED INTERMITTENCY}

At the collision the chaotic attractor loses its stability and is converted into a BCS. The SCS is robust and persists after the crisis. Figure 6(c) shows these two non-attracting chaotic sets at $\alpha=2.85523 \pi$, after the interior crisis. Both the BCS and the SCS display the characteristic gaps that reflect the fractal structure of chaotic saddles along their unstable direction. ${ }^{2}$ After the onset of the interior crisis, an infinite number of coupling UPOs are created, filling the gap regions via an event called explosion. ${ }^{25,26}$ These newly created unstable periodic orbits have components in both surrounding and banded regions and are responsible for the coupling of the two regions. In general, the coupling UPOs are difficult to find numerically, due to their long periods. This is a particularly complex task in a spatially extended system. To this end we adapted the method of Lathrop and Kostelich, ${ }^{27}$ originally used to search for UPOs in experimental chaotic time series. First, we get a time series containing 250000 points $\mathbf{z}_{k}$ of the two-dimensional Poincaré map projection described in Sec. IV. Then, the following distance function is defined

$$
d(k, p)=\left\|\mathbf{z}_{k}-\mathbf{z}_{k+p}\right\|,
$$

where $p$ is the specified period of the UPO to be found. When $d(k, p)<\epsilon, \mathbf{z}_{k}$ is called a recurrent point. We set $\epsilon=10^{-6}$

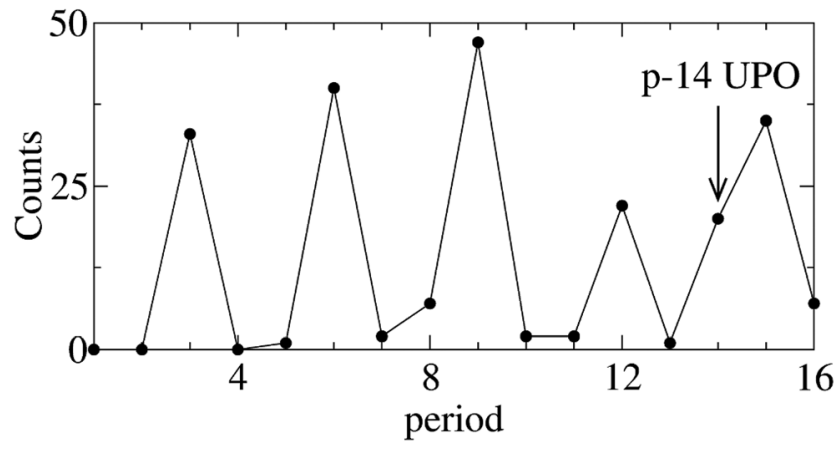

FIG. 7. Histogram of recurrent points for a Poincaré time series at $\alpha=2.85523 \pi$. UPOs with period multiple of 3 are clearly detected. The first peak not being a multiple of 3 corresponds to a $p-14$ UPO, marked by an arrow.

which defines the accuracy level of the UPO detection. A recurrent point is not necessarily a component of a periodic orbit of period $p$. However, if a recurrent period $p$ appears frequently, it is likely that the corresponding recurrent points are close to periodic orbits of period $p$. The idea is to construct a histogram of the recurrent points for each period and identify peaks in the histogram. Figure 7 shows the histogram for the Poincaré time series at $\alpha=2.85523 \pi$. It clearly shows the peaks corresponding to the different UPOs of period multiple of 3 created within the periodic window at each period doubling bifurcation. The first peak at a period not being a multiple of 3 is $p=14$. By inspecting the recurrence points for $p=14$ we see that they occupy almost exactly the same place in the phase space. We identify the set of recurrent points as a p-14 coupling UPO, which is plotted as green crosses in Fig. 6(c). The spatio-temporal dynamics of electron density associated to this coupling UPO is shown in Figs. 8(a) and 8(b) shows the corresponding time series for $x=0.5$. We see that the first temporal minimum at $x=0.5$, appearing at $t \approx 3.2$ (left arrow in Fig. 8(b)) after having passed through other thirteen local minima, reappears at $t \approx 61.6$, marked by the right arrow in Fig. 8(b). The relation of the UPO dynamics with the chaotic dynamics of the Pierce diode for the control parameter

(a)

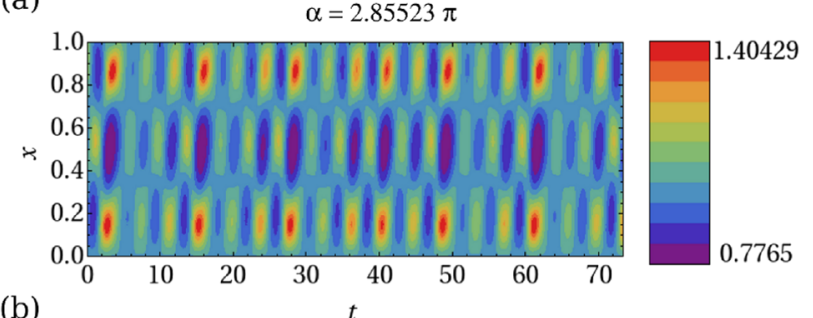

(b)

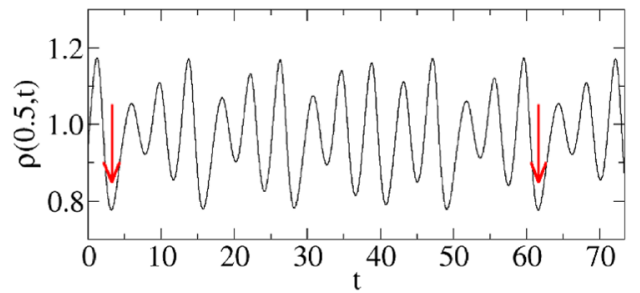

FIG. 8. (a) Spatio-temporal evolution of the electron density of the $p-14$ UPO. (b) Temporal evolution of the electron density at $x=0.5$ in the same time interval as (a). The time interval between two red arrows corresponds to one period of the UPO. 


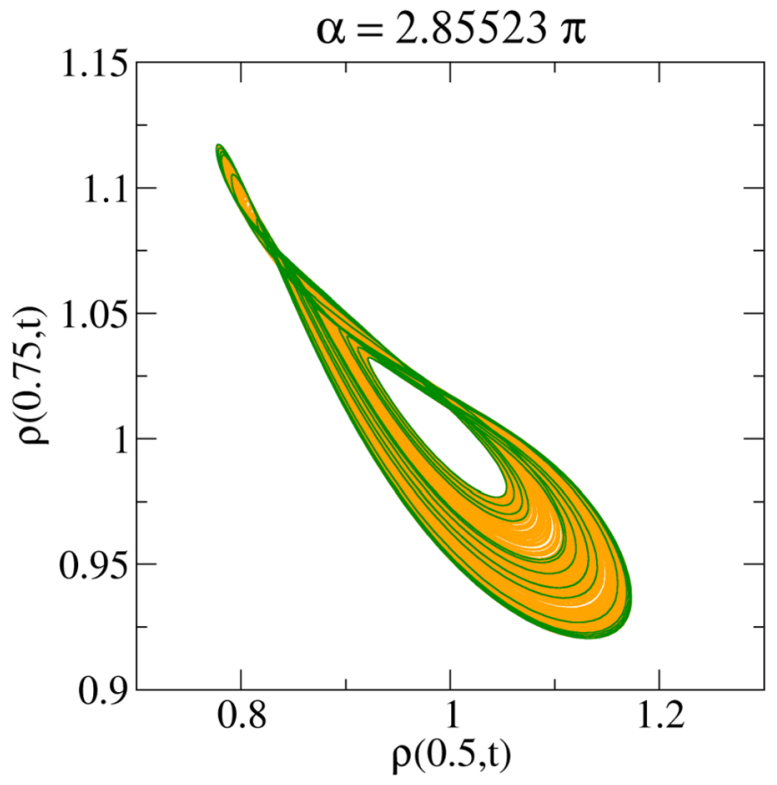

FIG. 9. Two-dimensional projection of the continuous time evolution of the $p$-14 coupling UPO (green curve) and the chaotic attractor (orange area).

$\alpha=2.85523$ is illustrated in Fig. 9, showing the continuous time evolution of the electron density at $x=0.5$ and $x=0.75$ for the $p$-14 UPO (green curve) and the chaotic attractor of the system (orange area). We see that the coupling UPO trajectory is nicely embedded in the orange area, thus appearing as a subset of the attractor.

An enlargement of the same region of phase space as in Fig. 6(b) is shown in Fig. 6(d), including the edge state (black cross) and its stable manifold (dashed lines). After the crisis the edge of chaos separates the two regions occupied by SCS and BCS.

The link between both regions provided by the coupling UPOs has, as a direct consequence, the formation of a larger chaotic attractor characterized by an intermittent behavior. Figure 10 shows the time series of crisis-induced intermittency ${ }^{28}$ corresponding to the post-crisis chaotic attractor. The laminar and bursty periods of the crisis-induced intermittency correspond to the trajectory visiting the vicinity of the BCS and SCS chaotic saddles, respectively. Grebogi et al. ${ }^{28}$ showed that the characteristic intermittency time $\tau$, obtained as the average over a long time series of the time between bursty periods, follows the scaling law

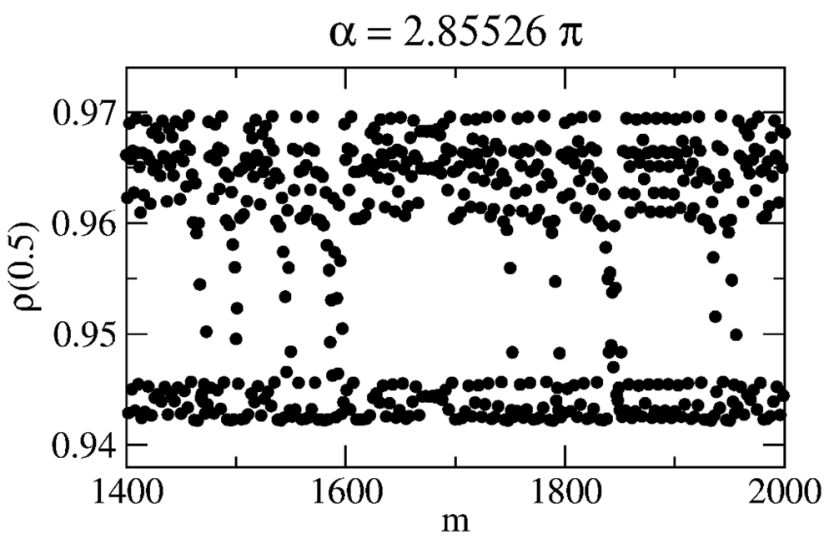

FIG. 10. Poincaré time series showing crisis-induced intermittency.



FIG. 11. Characteristic intermittency time $\tau$ as a function of $\alpha_{C}-\alpha$ in log$\log$ scale. The red line is the linear fit of the values of the intermittency time computed from time series (black dots).

$$
\tau=\left(\alpha_{\mathrm{C}}-\alpha\right)^{-\gamma}, \quad \alpha \lesssim \alpha_{\mathrm{C}}
$$

where $\gamma$ is the critical exponent. In Fig. 11 we plot $\tau$ as a function of $\alpha_{C}-\alpha$ in log-log scale. The linear behavior is consistent with the power law Eq. (13), which critical exponent $\gamma=0.56 \pm 0.02$ is obtained from the slope of the linear fit represented by the red line.

\section{CONCLUSION}

In this work the edge of chaos, a structure that separates the transient behavior from the attracting one is examined in the Pierce diode for the first time. We showed that in a periodic window of that system, the edge state is the $p-3$ unstable periodic orbit that emerges from a saddle-node bifurcation at the start of the periodic window. In addition, we obtained the direct evidence of the crucial role of the edge of chaos in an interior crisis, a ubiquitous chaotic transition at the end of periodic windows. From this direct evidence, it is expected that the dynamical properties of chaotic transitions are defined by the edge of chaos and the edge state. This is a significant finding since it can be used to understand more complex transitions in high-dimensional dynamical systems. ${ }^{3,29,30}$

\section{ACKNOWLEDGMENTS}

This work was supported by CAPES, CNPq, and FAPESP Brazilian Agencies. ACLC acknowledges the award of a Marie Curie International Incoming Fellowship and the kind hospitality of Paris Observatory.

${ }^{1}$ J. D. Skufca, J. A. Yorke, and B. Eckhardt, "Edge of chaos in a parallel shear flow," Phys. Rev. Lett. 96, 174101 (2006).

${ }^{2}$ E. L. Rempel, A. C.-L. Chian, E. E. N. Macau, and R. R. Rosa, "Analysis of chaotic saddles in high-dimensional dynamical systems: The KuramotoSivashinsky equation," Chaos 14, 545-556 (2004).

${ }^{3}$ E. L. Rempel and A. C.-L. Chian, "Origin of transient and intermittent dynamics in spatiotemporal chaotic systems," Phys. Rev. Lett. 98, 014101 (2007).

${ }^{4}$ E. L. Rempel, G. Lesur, and M. R. E. Proctor, "Supertransient magnetohydrodynamic turbulence in keplerian shear flows," Phys. Rev. Lett. 105, 044501 (2010). 
${ }^{5}$ T. M. Schneider, B. Eckhardt, and J. A. Yorke, "Turbulence transition and the edge of chaos in pipe flow," Phys. Rev. Lett. 99, 034502 (2007).

${ }^{6}$ P. A. Cassak, J. F. Drake, M. A. Shay, and B. Eckhardt, "Onset of fast magnetic reconnection," Phys. Rev. Lett. 98, 215001 (2007).

${ }^{7}$ J. Vollmer, T. M. Schneider, and B. Eckhardt, "Basin boundary, edge of chaos and edge state in a two-dimensional model," New J. Phys. 11, 013040 (2009).

${ }^{8}$ J. R. Pierce, "Limiting stable current in electron beams in the presence of ions," J. Appl. Phys. 15, 721 (1944).

${ }^{9}$ B. B. Godfrey, "Oscillatory nonlinear electron flow in a Pierce diode," Phys. Fluids 30, 1553-1560 (1987).

${ }^{10}$ W. S. Lawson, "The Pierce diode with an external circuit. I. Oscillations about nonuniform equilibria," Phys. Fluids 1, 1483-1492 (1989).

${ }^{11} \mathrm{M}$. Hörhager and S. Kuhn, "Weakly nonlinear steady-state oscillations in the Pierce diode," Phys. Fluids B 2, 2741-2763 (1990).

${ }^{12}$ D. I. Trubetskov, E. S. McHedlova, V. G. Anfinogentov, V. I. Ponomarenko, and N. M. Ryskin, "Nonlinear waves, chaos and patterns in microwave electronic devices," Chaos 6, 358-367 (1996).

${ }^{13}$ G. S. Nusinovich, Y. Carmel, T. M. Antonsen, D. M. Goebel, and J. Santoru, "Recent progress in the development of plasma-filled traveling-wave tubes and backward-wave oscillators," IEEE Trans. Plasma Sci. 26, 628-645 (1998).

${ }^{14}$ D. M. Goebel, E. S. Ponti, R. L. Eisenhart, and R. W. Lemke, "Frequency and power response of high-power plasma-filled backward-wave oscillators," Phys. Plasmas 6, 2319-2322 (1999).

${ }^{15}$ Y. P. Bliokh and G. S. Nusinovich, "Nonlinear theory of beam-wave interaction in the pasotron with a phase-mixed electron beam," Phys. Plasmas 13, 023102 (2006).

${ }^{16}$ R. A. Filatov, A. E. Hramov, Y. P. Bliokh, A. A. Koronovskii, and J. Felsteiner, "Influence of background gas ionization on oscillations in a virtual cathode with a retarding potential," Phys. Plasmas 16, 033106 (2009).

${ }^{17}$ J. J. Barroso, M. O. Terra, and E. E. N. Macau, "Bifurcation and chaos in the second oscillatory window of the classical Pierce diode," Int. J. Bifurcation Chaos Appl. Sci. Eng. 11, 2579-2586 (2001).
${ }^{18}$ R. A. Treumann and W. Baumjohann, Advanced Space Plasma Physics (Imperial College Press, London, 1997).

${ }^{19}$ A. E. Hramov, A. A. Koronovskii, and I. S. Rempen, "Controlling chaos in spatially extended beam-plasma system by the continuous delayed feedback," Chaos 16, 013123 (2006).

${ }^{20} \mathrm{H}$. Matsumoto, H. Yokoyama, and D. Summers, "Computer simulations of the chaotic dynamics of the Pierce beam-plasma system," Phys. Plasmas 3, 177-191 (1996).

${ }^{21}$ G. Benettin, L. Galgani, A. Giorgilli, and J.-M. Strelcyn, "Lyapunov characteristic exponents for smooth dynamical systems and for Hamiltonian systems - A method for computing all of them. I - Theory. II - Numerical application," Meccanica 15, 9-30 (1980).

${ }^{22}$ C. Grebogi, E. Ott, and J. A. Yorke, "Crises, sudden changes in chaotic attractors, and transient chaos," Physica D 7, 181-200 (1983).

${ }^{23}$ A. C.-L. Chian, W. M. Santana, E. L. Rempel, F. A. Borotto, T. Hada, and Y. Kamide, "Chaos in driven Alfvén systems: Unstable periodic orbits and chaotic saddles," Nonlinear Processes Geophys. 14, 17-29 (2007).

${ }^{24}$ G.-H. Hsu, E. Ott, and C. Grebogi, "Strange saddles and the dimensions of their invariant manifolds," Phys. Lett. A 127, 199-204 (1988).

${ }^{25}$ C. Robert, K. T. Alligood, E. Ott, and J. A. Yorke, "Explosions of chaotic sets," Physica D 144, 44-61 (2000).

${ }^{26}$ K. G. Szabó, Y.-C. Lai, T. Tél, and C. Grebogi, "Topological scaling and gap filling at crisis," Phys. Rev. E 61, 5019-5032 (2000).

${ }^{27}$ D. P. Lathrop and E. J. Kostelich, "Characterization of an experimental strange attractor by periodic orbits," Phys. Rev. A 40, 4028-4031 (1989).

${ }^{28}$ C. Grebogi, E. Ott, F. Romeiras, and J. A. Yorke, "Critical exponents for crisis-induced intermittency,” Phys. Rev. A 36, 5365-5380 (1987).

${ }^{29} \mathrm{~K}$. He and A. C.-L. Chian, "On-off collective imperfect phase synchronization and bursts in wave energy in a turbulent state," Phys. Rev. Lett. 91, 034102 (2003).

${ }^{30}$ A. C.-L. Chian, R. A. Miranda, E. L. Rempel, Y. Saiki, and M. Yamada, "Amplitude-phase synchronization at the onset of permanent spatiotemporal chaos," Phys. Rev. Lett. 104, 254102 (2010). 